Im 1. Teil wurde versucht, den Begriff Stereotyp zu definieren, wobei Aspekte der Stereotypenforschung eingehender betrachtet wurden. Der 2. Teil beschäftigt sich nun mit Theorien und Untersuchungen zur Entstehung von Stereotypen, wobei die sogenannten Stereotypensysteme und deren Funktionen mit herangezogen werden.

\section{Zur Enstehung von Stereotypen}

In der Sozialpsychologie geht man davon aus, dass der Sozialisierungsprozess einzelner Individuen in einer Gruppe die folgenden Aspekte menschlichen Verhaltens einschließt: Motivation, Lernen und Wahrnehmung, wobei alle drei Gebiete voneinander ahängen. In Bezug auf das Lernen geht man zunächst auch von drei Hauptgebieten aus, die zum Sozialisierungsprozess gehören: Sehen lernen, Handeln lemen und Lernen der Gefühlssteuerung. Das Sehen-lernen bezieht sich auf die Art der Strukturierung der Wahrnehmungswelt durch die Teilhabe am Leben einer bestimmten Gruppe. Das Handeln-Lernen ist gruppenspezifisch. Das einzelne Individuum lernt das, was von inm verlangt wird und passt sich an die Erfordernisse einer jeweiligen Gruppe an. Das Zusammenspiel von individuellen Bedürfnissen und den sozialen Forderungen und den daraus resultie- der Gruppe wird durch den Lernprozess so gelernt, dass das einzelne Individuum Gegenstände und Ereignisse so wahrnimmt, wie es die restlichen Mitglieder dieser Gruppe tun. Die Wahrnehmung istalso eine erlernte Reaktion auf innere und äußere Reize. Sie unterliegt sozialen Einflüssen und bestimmt auch das soziale Verhalten. Die jeweilige Wahrnehmungsstruktur eines Individuums stellt seine wiederholte Begegnung mit der Welt dar. Dabei spielt die Kommunikation eine große Rolle, denn ohne sie gibt es keine Gruppenteilhabe. Das einzelne Individuum, also der Mensch, braucht Worte und Gesten als Verständigungsmittel und weist sich somit als Angehöriger einer jeweiligen Gruppe aus. Wichtig ist dabei, welche Wirksamkeit die Verwendung oder Nichtverwendung von Zeichen und Symbolen hat. Die Kommunikation, durch die der Mensch auf den Anderen wirkt, ist der eigentliche Träger des sozialen Geschehens. Erst durch sie wird der Mensch zum sozialen Wesen in einer Gruppe oder einer Kultur und kann sich in ihr als solcher behaupten.

Das Bestehen einer jeweiligen Kultur ist eigentlich auf die zwischenmenschliche Kommunikation gegründet. Ohne Kommunikation könnten sich die Menschen nicht zusammenschließen, und es gäbe keine kulturelle Teilhabe. Innerhalb einer Kultur werden Normen, kulturelle Überlieferungen und traditionelle Wertvorstellungen von dieser vorgeprägt und stereotypisiert. Diese Werte, Normen und Formen innerhalb einer Kultur werden als normal und

\title{
DAS FREMDE UND DAS $\mathbb{E}$ GENE: $S$ EREOTYPEN UND \\ FREMDSPRACHENUNTE RICHT (III)
}

renden Ergebnissen wird als das Lernen der Gefühlssteuerung bezeichnet.

Sozialisierung ist ein fortgesetztes Lernen. Durch den Sozialisierungsvorgang wird aus dem undifferenzierten Organismus ein Individuum (Hartley, 1969). Hierbei ist die Aneignung einer spezifischen Auffassung von Ich und Umwelt ein wesentlicher Bestandteil, da hier die Grundlage jeder Gruppenzugehörigkeit liegt. Innerhalb natürlich angesehen, "(...) weil sie ja eingefahren sind als die festen Engramme alltagskultureller Orientierung, und dass vor diesem Hintergrund das Abweichende, Ungewöhnliche registriert wird". (Bausinger, 1988)

Grundlage der Stereotypisierung ist hier ein von kognitiven Regeln gesteuerter Prozess. Diese Regeln oder Schemata sind tatsächliche oder vermeintliche Zusammenhänge zwischen Ereignissen, Merkmalen oder Reizen in der Umwelt. Nach Stapf, Stroebe und 
Jonas (1986) lassen sich die soziokulturellen Bedingungen für die Entstehung von Stereotypen systematisch in fünf Teilaspekte unterscheiden:

\section{Gruppenbildungen und Gruppenkonflikte \\ 2. Schicht und Bildung \\ 3. Soziokulturelle Normen \\ 4. Sozialisation \\ 5. Bezugsgruppen}

In Bezug auf Gruppenbildungen und Gruppenkonflikte beziehen sich diese auf die zwischen nationalen und ethnischen Gruppen bestehenden politischen, wirtschaftlichen und sozialen Beziehungen. Diese Beziehungen untereinander erzeugen ihrer Meinug nach gegenseitig Stereotype. Der 2. Teilaspekt (Schicht und Bildung) besagt, dass Mitglieder höherer Gesellschaftsschichten aufgrund ihrer Bildung und ihres Standes eher weniger negative Stereotype und Einstellungen gegenüber einer Minorität oder einer anderen ethnischen Gruppe haben als Angehörige niederer Schichten. Diese These wird von innen aber gleichzeitig als nicht allgemeingültig dargestellt, da es aufgrund des Forschungsstandes keine eindeutige Erklärung des Zusammenhanges von Stereotypisierung und Schichtzugehörigkeit gibt. Ebenso wirken sich die soziokulturellen Normen (3.Teilaspekt) auf die Entstehung und Entwicklung von Stereotypen aus. Die Sozialisation (4. Teilaspekt) ist der Prozess, in dem ein Kind die Überzeugungen, Normen und Werte seiner Kultur übernimmt. Als 5. Teilaspekt werden die Bezugspersonen genannt. Das Individuum teilt mit einer Gruppe (Bezugsgruppe) Werte und Einstellungen. Bei der Stereotypenbildung handelt es sich also primär um ein sozialpsychologisches Problem.

Quasthoff (1973), die u.a. den sprachwissentschaftlichen Aspekt diesbezüglich untersucht, befasst sich entsprechend mit der Entstehung von Stereotypen. Sie geht allerdings von der "Übernahme von sozialen Vorurteilen" (Quasthoff, 1973) aus. Ihrer Meinung nach ist das soziale Vorurteil Gegenstand der Sozialpsychologie, der Soziologie und der Psychologie; die Überzeugung ist Gegenstand der Psychologie und der Sozialpsychologie, hingegen ist das Stereotyp Gegenstand der Linguistik. Wobei sich Sozialpsychologie und Psychologie mit der Entstehung von Vorurteilen und Stereotypen befassen.
Die Annahme, dass Stereotype oder soziale Vorurteile angeboren seien, ist also falsch. Sie werden nicht individuell vererbt, sondern gelernt, bzw. entwickeln sich in den Lern- und Adaptionsmechanismen. Ergebnisse in der Vorurteilsforschung haben ergeben, dass Vorurteile bei Kindern ab dem 4. - 5. Lebensjahr entstehen können, genau zu dem Zeitpunkt, in dem sich nach der Freudschen Theorie durch Identifikation mit den Eltern beim Kind das Über-Ich herausbildet. Erste Erfahrungen mit sozialen Regeln, die das Kind macht, vollziehen sich in der Familie als Primärgruppe. Hier lernt es sich entsprechend zu verhalten, wobei der Lernprozess von den jeweiligen kulturellen und den sozialen Konventionen bestimmt wird. Außerfamiliäre Kontakte erweitern den Lernvorgang durch Äußerungen, Haltungen und Gebärden oder bestimmte Verhaltensweisen. Das Kind lernt, sich den Maßstäben der Eltern entsprechend zu verhalten und darüber hinaus auch das Fremde, das von außen auf die gewohnte Umgebung Wirkende entsprechend zu bewerten. Durch die Eltern werden die ersten Sozialisierungsprozesse durchgeführt. Sie vermitteln dem Kind die Normen und Verhaltensweisen der jeweiligen Kultur, in der sie integriert sind. Hier werden auch die ersten Stereotype vermittelt, wie z.B. die abwertenden Stereotypen gegen das Fremde und das

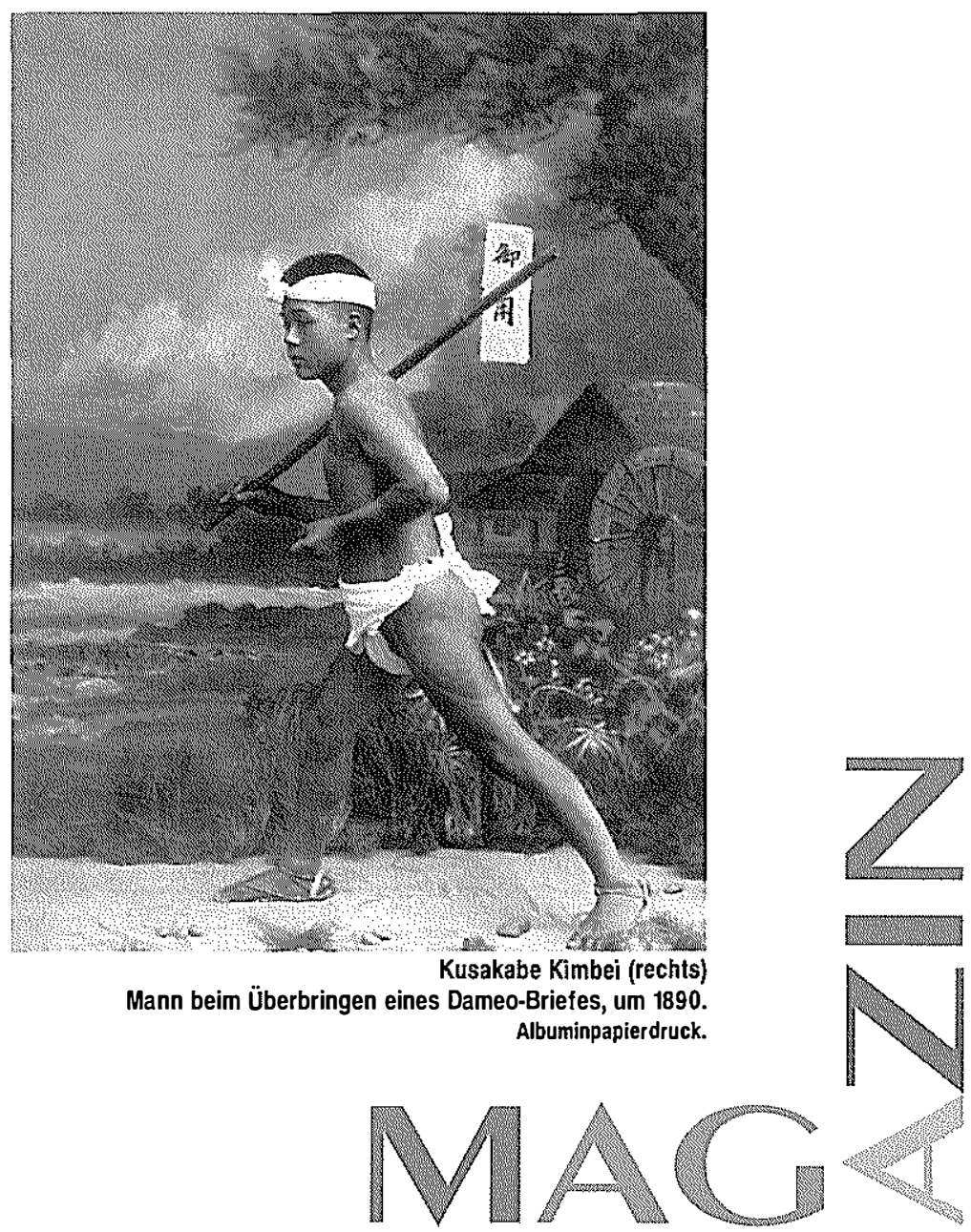


Andersartige, die mit Eigenschaften besetzt sind (z.B. Faulheit, Dummheit und Verlogenheit), die das Kind nicht übernehmen soll, sowie auf der anderen Seite die befürwortenden, positiven Stereotypen, nach denen die Eltern und die jeweilige Gruppe leben. Das Bekannte und das Freundliche beinhalten entsprechende Eigenschaften (Fleiß, Klugheit, Ehrlichkeit), die auch das Kind lernen und übernehmen soll. Nach Quasthoff beginnen die Kinder spätestens im Alter von 9 Jahren, die Muster von Stereotypen zu übernehmen und zu entwickeln, die ihnen zunächst die Eltern und deren soziales Umfeld vorgeben. Die Schule, in die das Kind aufgenommen wird, ist die nächste Instanz. Hier wird ein großer Teil von sozialen Einstellungen bzw. Stereotypen vermittelt. Die im Familienumfeld gemachten Erfahrungen mit dem Fremden sind hier noch individuell, während sie in der Schule für ganze Volksgruppen generalisiert werden. Die Wissensvermittlung findet oft nicht nach rein objektiven Sachgesichtspunkten statt. Automatisch fließen hier die Normen und Stereotype einer jeweiligen Zugehörigkeitsgruppe mit ein und können das Stereotypensystem der Schüler entsprechend prägen. "Elternhaus und Schule sind formelle Gebilde mit festen Erziehungszielen. Daneben sind Kinder und Erwachsene Mitglieder informeller Gruppen". (Dröge, 1967).

Informelle Gruppen, wie z. B. Arbeitskollegen oder Hausfrauen, die sich beim Einkaufen treffen, gelten hier ebenso als Vermittler von Stereotypen.

Wertvorstellungen, Normen und andere kulturelle Überlieferungen werden in der Form wahrgenommen, die die jeweilige Kultur entsprechend vorgeprägt und stereotypisiert hat. Ein wesentlicher Faktor, der den Lernprozess begründet, ist das Bedürnis des Menschen nach sozialer Realität. Das einzelne Individuum einer Gruppe hat das Bedürfnis nach Informationen und ist davon abhängig, da es sich aus der Summe dieser Informationen eine vorgestellte Realität auf-

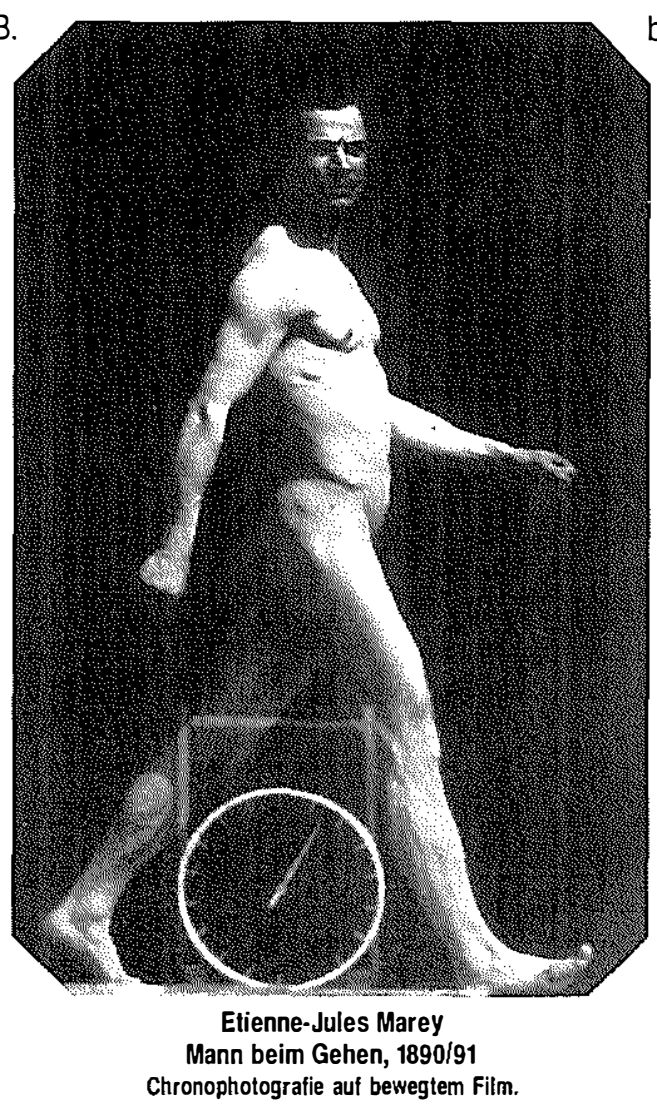

bauen muss, weil es diese nicht selbst gelebt und erfahren hat.

Zusammenfassend soll hier zur Theorie der Stereotypisierung Lilli (1982) genannt werden, der u.a. davon ausgeht, "(...) dass die Bildung von Urteilen ein von kognitiven Regeln gesteuerter Prozess ist. Die Regeln beinhalten Annahmen über das gemeinsame Auftreten von Variablen in der Umwelt. Je nach der Art der Kovariation der Umweltvariablen können in dem resultierenden Urteil systematisch Verzerrungen enthalten sein, die entweder durch überbetonte Differenzierung oder durch überbetonte Klassifizierung von Reizen zustande kommen. Als Stereotypisierungseffekte werden die systematischen Urteilsverzerrungen verstanden, die durch Überbetonung der Klassifizierung von Reizen entstehen". Für ihn enthält Stereotypisierung dementsprechend vier zentrale Punkte:

"1. Die Annahme der Kovariation von Umweltreizen

2. Die daraus resultierenden Effekte der Umweltverzerrung und

3. Deren reziproke Beziehung sowie

4. Die Konsistenz der resultierenden Reize."

In der Stereotypenforschung werden bei den Untersuchungen zu einer möglichen Entstehung von Stereotypen auch einzelne Stereotypensysteme und deren Funktionen herausgestellt. An dieser Stelle sollen hier einige kurz genannt werden, da durch die Thematisierung und Gegenüberstellung in der Forschungsliteratur weitere Aspekte zur Entstehung von Stereotypen erkennbar sind, bzw. werden. Dabei gibt es theoretische Ansätze, die sich mit denen in den vorangegangenen Abschnitten vorgestellten in inren Grundsatzfragen und -theorien überschneiden können, im folgenden teilweise aber erneut angesprochen werden, um so eine bessere Strukturierung erkennbar zu machen.

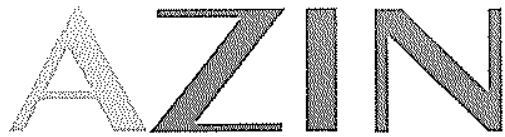




\section{Stereotypensysteme und Funktionen}

Ein einheitliches Stereotypenkonzept ist in der Forschungsliteratur nur schwer erkennbar. Vielmehr ist oft eine zunehmende Aufsplitterung der Termini zu sehen. Die wissenschaftliche Auseinandersetzung mit Stereotypen versucht nach wie vor, sich an der Realität zu orientieren, um so zu einer adäquaten wissenschaftlichen Analyse zu gelangen. Hierbei ist es unumgänglich, die Funktionen von Stereotypen und Vorurteilen zu betrachten und, wie bereits mehrfach versucht, Stereotype in entsprechende Systeme einzuteilen, um so den Gegenstand als solchen differenziert erfassen zu können. Lilli geht entsprechend von drei unterschiedlichen Stereotypensystemen aus:

1. Stereotype als Orientierungssysteme

2. Stereotype als Anpassungssysteme und

3. Stereotype als Aufrechterhaltung des Selbst (Lilli, 1982).

Das Stereotyp als Orientierungsfunktion beinhaltet, dass die Bilder, die wir in unserem Kopf haben, die Orientierung erleichtern. Schlüsselreize genügen, um fertige Bilder hervorzuheben. Die Wirklichkeit wird kategorisiert. Automatisch werden vereinfachte Entscheidungskategorien bereitgestellt, die aus einer narürlichen Ökonomie in einer jeweiligen Kultur entstanden sind. Hierzu Bausinger (1988): "Stereotype ordnen diffuses Material und reduzieren Komplexität; darin liegt eine wichtige Orientierungsfunktion".

In Bezug auf die Stereotype als Anpassungssysteme und als Identifikationsfunktion sind die o.g. Bilder in unserem Kopf überwiegend kulturspezifisch und beziehen sich auf eine bestimmte Gesellschaft oder Gruppe. In Bezug auf das Individuum entstehen hier Stereotype durch die Versuche, Belohnung zu erhöhen und Bestrafungen zu verringern. Viele Individuen einer Gruppe haben bestimmte Stereotype nur deshalb, weil sie innen das Leben in dieser Gruppe erleichtern. Sie tragen u.a. zur Verminderung von Spannungen und Konflikten bei. Das Individuum passt sich einer jeweiligen Gruppe an und identifiziert sich mit inr (Identifizierungsfunktion). Dabei werden im Anpassungsvorgang Einstellungen und Überzeugungen übernommen. Gleiche oder ähnliche Einstellungen können somit 0.g. Spannungen und Konflikten innerhalb einer Gruppe verringern bzw. teilweise gar nicht entstehen lassen. Durch Anpassung erfährt das einzelne Individuum Bestätigung in der jeweiligen Gruppe.
Stereotypie wird hier als Mittel zur Identifikation gesehen. Bei den Stereotypen als Systemen zur Aufrechterhaltung des Selbst dienen die Bilder in unserem Kopf der Selbstverankerung in einer jeweiligen Gruppe und erleichtern somit die Umweltorientierung und das Zusammenleben. Stereotype werden hier als Perspektiven gesehen, die vorgegeben sind und zur Beibehaltung des Selbst wichtig sein können. Ein Individuum braucht für seine eigene Stabilität Bezugspunkte, um die Wirklichkeit und Umwelt innerhalb einer Gruppe entsprechend erleben zu können (Selbstbehauptungs- und Stabilisierungsfunktion). Dabei spielt es eine wichtige Rolle, inwieweit ein bestimmtes Stereotyp beibehalten wird, wenn es als Bestandteil des Selbst zentral ist. Ist die eigene Selbstsicherheit in Gefahr, z.B., wenn die Umwelt im bekannten Sinne nicht mehr oder nur teilweise vorhanden ist und somit neu definiert werden muss, wird die Stabilität des Selbst eines Individuums durch die Übernahme von Stereotypen und Vorurteilen wieder hergestellt. Bergler (1976) spricht hier von "notwendigem Ausfüllen von Leer-Räumen". Diese Leer-Räume können Orientierungslosigkeit, verbunden mit Unsicherheit und Bedrohung der Person, auslösen. Die daraus resultierende Angst kann jedoch dadurch überwunden werden, indem eine neue Situation (eine neue Umwelt) kategorisiert und klassifiziert wird. Dies geschieht auch entsprechend durch die Übernahme von Stereotypen und Vorurteilen. Aus psychoanalytischer Sichtweise werden Stereotypen und Vorurteile als Antworten auf die eigenen innerer Konflikte angesehen.

Bausinger (1988) geht bei seinen theoretischen Ansätzen zur Definition von Stereotypen u.a. auch davon aus, was Stereotype leisten, um so Mängel wie Übergeneralisierung, Erstarrung und Immunisierung nachzuweisen. Er hebt drei "Leistungen" hervor:

1. Stereotype entstehen nicht immer, aber in der Regel aus Überverallgemeinerung tatsächlicher Merkmale; es ist innen also ein relativer Wahrheitsgehalt zuzusprechen.

2. Stereotype ordnen diffuses Material und reduzieren Komplexität; darin liegt eine wichtige Orientierungsfunktion.

3. Stereotype bieten Identifikationsmöglichkeiten an, über die neue Realbezüge entstehen können; es ist also mit einer realitätsstiftenden Wirkung von Stereotypen zu rechnen.

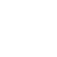


Hiernach ist Stereotypie kein defizienter Versuch sondern ein Erfahrungsmodus. Erkenntnisse, die ordnend, benennend und klassifizierend sind, schließen folglich die Stereotypisierung nicht aus. Nicht nur Personen und Tiere, sondern auch Vorgänge und Konstellationen werden mit entsprechenden charakterisierenden und neutralisierenden Etiketten versehen, um so identifiziert zu werden. Sie werden benannt und die anschließende Erkennung bekommt ihre jeweilige Form, die wiederum das Erkennen strukturiert und begrenzt.

An dieser Stelle soll ein weiterer Funktionskomplex genannt werden, der nach Quasthoff zum Bereich der

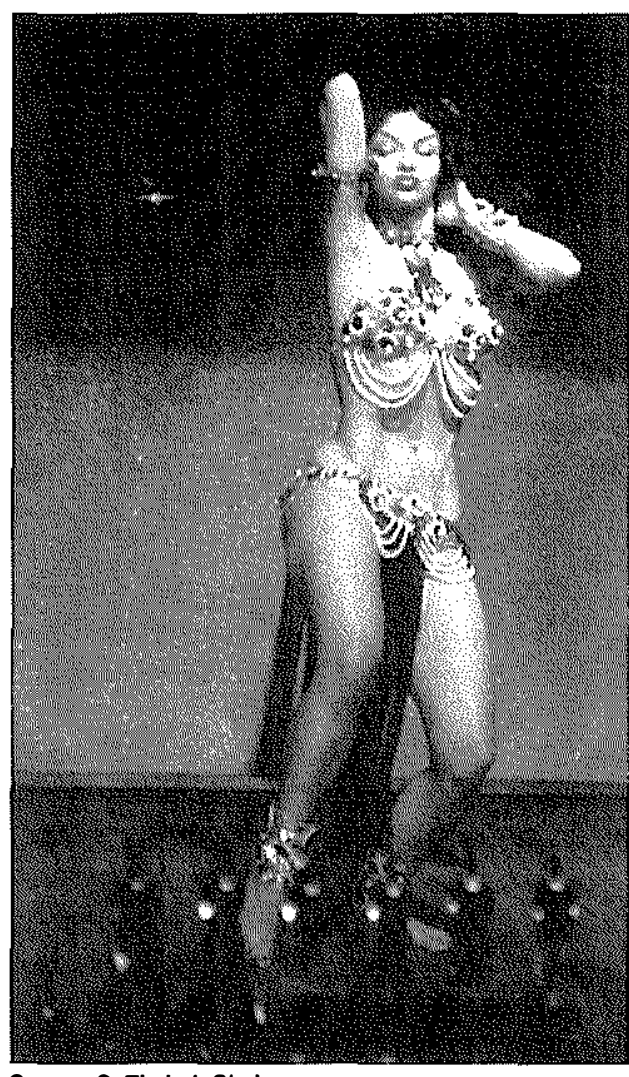

Funktion sozialer Vorurteile gehört, jedoch nach Stapf, Stroebe und Jonas (1986) unter den motivationalen Bedingungen zur Entstehung von Stereotypen angesiedelt ist. Hierbei handelt es sich um die Rechtfertigungsfunktion bzw. den "SündenbockMechanismus". Innerhalb einer jeweiligen Gruppe wird das Individuum oft mit bestimmten Frustrationen konfrontiert. Diese entste-hen durch eigene unpersönliche Frustrations-quellen (z.B. wirtschaftliche Krisen, soziale Benachteiligung) oder durch andere Individuen. Diese Frustrationen haben zur Folge, dass nach entsprechenden Sündenböcken gesucht wird, um die erlittenen Frustrationen durch negative Einstellungen oder George S. Zimbel. Chelo, Bourbon Street, New Orleans, 1955. Gelatine-Silberdruck.

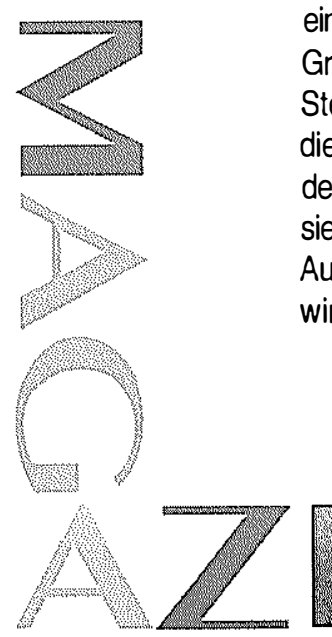
Aggressivität abzureagieren und zu rechtfertigen. Ist der Verursacher einer Frustration zu mächtig oder nicht identifizierbar, so werden aggressive Tendenzen auf eine Außengruppe oder einzelne Mitglieder dieser Gruppe gerichtet. Dabei übernehmen sie die Stellvertretung für den Verursacher der Frustration. Für diese Autoren ist die Sündenbock-Theorie als Theorie der Entwicklung von Stereotypen eher unvollständig, da sie nicht erklären kann, warum eine bestimmte Außengruppe oder ein bestimmtes Mitglied ausgewählt wird. Man kann lediglich davon ausgehen, dass
Sündenböcke oft aus Rand- und Minoritätsgruppen ausgewählt werden, da sie relativ machtlos erscheinen. Diese Frustration-Aggressions-Hypothese hat u.a. auch zur Folge, dass in Krisenzeiten mit politischen und wirtschaftlichen Problemen der Rassenhass zunimmt. Abschließend sei hier folgendes Zitat genannt: "Der Sündenbock-Mechanismus" funktioniert, indem die Schuld für unsere Schwierigkeiten (Wessen? Welche?) auf die Fremdgruppe gelenkt wird". (Quasthoff, 1973)

\section{Schlussfolgerungen}

Geht man in der Interkulturellen Kommunikation von der Zielsetzung aus, interkulturell-landeskundliche Inhalte zu vermitteln, und gilt interkulturelle Landeskunde als Voraussetzung interkultureller Kommunikation, so bietet die aktive Verwendung von Unterrichtsmaterialien landeskundlichen Inhalts zum Zwecke einer interkulturell orientierten Begegnungsdidaktik vielseitige Möglichkeiten, dieser Zielsetzung zu entsprechen. Durch ihren Einsatz können den Lernenden Land und Leute der Zielsprache nähergebracht, Unterschiede und mögliche Gemeinsamkeiten sichtbar gemacht werden. Bei ihrer Verwendung im DaF-Unterricht ist von einer realitätsbezogenen Darstellung auszugehen. Wenn hier z.B. kultur- bzw. landesspezifische Gewohnheiten zugrundegelegt werden, so sollten diese als mögliche, aber auch typische Eigenschaften von Menschen einer bestimmten Gesellschaft sichtbar werden. Die Zielsetzung sollte in der Darstellung eines Teils des deutschen Alltags liegen, um so Fremdes, sofern es als Fremdes verstanden wird, kennen- und verstehen $\mathrm{zu}$ lernen.

In den theoretischen Ansätzen zur Entstehung von Stereotypen geht man u.a. auch davon aus, dass eine Stereotypisierung nicht ausgeschlossen werden kann, wenn Erkenntnisse geordnet, benannt und klassifiziert werden. In internationalen Beziehungen und Kontakten kommt es sehr oft zu Einstellungkonzepten, die Stereotypen darstellen können. Ein Stereotyp kann also eine Einstellung oder Überzeugung sein, die generalisierend und wertend der Gesamtheit einer Gruppe "bestimmte Eigenschaften und/oder Verhaltensweisen zuoder abspricht". (Quasthoff, 1982). Bleiben bestimmte Einstellungen und Überzeugungen, kann man von einer Entstehung von Stereotypen ausgehen. Das Zusprechen einer Eigenschaft z.B.: "Die Deutschen sind kalt und unfreundlich" wird in der Stereotypenforschung 
als notwendige Funktion stereotyper Verallgemeinerungen gesehen. Kognitiv, affektiv oder sozial bestimmbare Funktionen von Stereotypen werden in einem gewissen Grad als harmlos, sogar als unverzichtbar angesehen. Das Verarbeiten bestimmter landeskundlicher Informationen kann oft nicht ohne Stereotypisierung funktionieren. Zusammenfassend soll hier noch einmal Quasthoff erwähnt werden, die herausstellt, dass die Linguistik und die (Sozial-) Psychologie aus heutiger Sicht Stereotype "d.h. unter den methodischen Rahmenbedingungen (...) eher als bestimme Form der Verarbeitung von Informationen (...) bzw. als bestimmte Wissensbestände (...) bezeichnen". (Quasthoff, 1988). Weiterhin beschreibt sie die kognitive Funktion von Stereotypen: "(Über-) Generalisierungen bei der Einordnung von Informationen - bei Beobachtungen zu Auffälligkeiten z.B. - aus der fremden Kultur stellen (...) einen durchaus vernünftigen interkulturellen Lernmechanismus dar".

Betrachtet man einen offenen und flexiblen Einsatz von Unterrichtsmaterialien, die landeskundliche Auswahlkriterien und damit verbundene sprachliche Lernziele entsprechend darstellen und verdeutlichen, stellt diese Material, wie wir längst wissen, eine Bereicherung im DaF-Unterricht dar, beachtet man dabei die ausreichende, ausdifferenzierte und ausführliche Didaktisierung und die Einhaltung der Zielsetzungen im Rahmen der Interkulturellen Kommunikation.

\section{Bibliographie:}

Allport, G.W.: Die Natur des Vorurteils, Köln 1971. Bausinger, Hans: Stereotype und
Wirklichkeit. In: Jahrbuch Deutsch als J.D. Fremdsprache, 14/1988, 157 - 170.

Buttjes, Dieter: Medien und Landeskunde, Zur Doppelfunktion der Medien im Fremdsprachenunterricht. In: Praxis des Neusprachlichen Unterrichts, Heft 2, 1982: 144 - 155.

Dröge, Franz W.: Publizistik und Vorurteil, Verlag Regensburg, Münster 1967.
Hofstätter, F.R.: Das Denken in Stereotypen, Vortrag, gehalten auf der Vortragsveranstaltung der Niedersächsischen Landesregierung zur Förderung der wissenschaftlichen Forschung in Niedersachsen, Heft 15.

Lilli, Waldemar: Grundlagen der Stereotypisierung, Verlag für Psychologie, Hagrete, Göttingen, Toronto, Zürich 1982.

Lippmann, Walter: Die öffentliche Meinung, Bochum 1990.

Manz, W:: Das Stereotyp, Zur Operationalisierung eines sozialwissenschaftlichen Begriffs, Meisenheim am Glan, 1968.

Quasthoff, Uta: Soziales Vorurteil und Kommunikation Eine Sprachwissenschaftliche Analyse des Stereotyps, Frankfurt am Main 1973.

Quasthoff, Uta: Sprachliche Bedeutung, soziale Bedeutung und soziales Handeln: Stereotype aus interkultureller Sicht, in: Müller, B.D. (Hg.), Konfrontative Semantik, Tübingen 1981: 75 - 93.

Quasthoff, Uta: Ethnozentrische Verarbeitung von Informationen: Zur Ambivalenz der Funktionen von Stereotypen in der interkulturellen Kommunikation, in: Matusche, P. (Hg.) Wie verstehen wir Fremdes? Dokumentation eines Werkstattgesprächs des GoetheInstituts München, iudicium, München 1989.

Stapf, K.H., Stroebe, W., Jonas, K.: Amerikaner über Deutschland und die Deutschen, Westdeutscher Verlag, Opladen, Darmstadt 1986.

Wenzel, A.: Stereotype in gesprochener Sprache, Form, Vorkommen und Funktion in Dialogen, Heutiges Deutsch, Reihe 1, Bd. 13, München 1978.

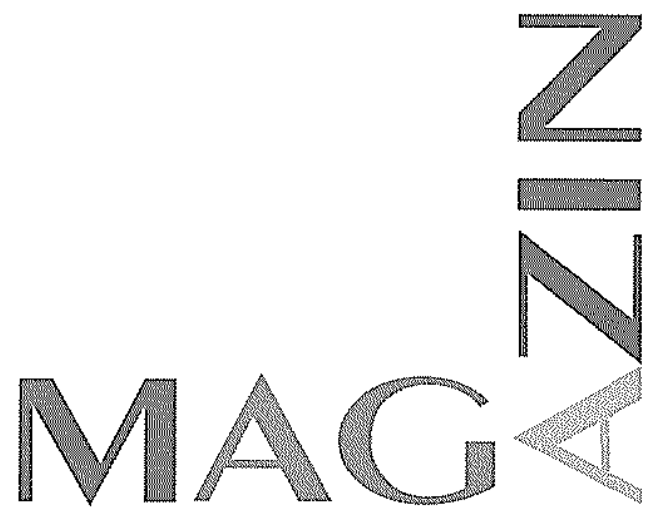

